\title{
A Strategy for Optimal Microgrid Operation Considering Vehicle-to-Grid Service
}

\author{
Van-Hai Bui, Akhtar Hussain and Hak-Man Kim ${ }^{1,2, *}$ \\ ${ }^{1}$ Incheon Nat'l University, Korea \\ ${ }^{2}$ Research Institute for Northeast Asian Super Grid, \\ Incheon Nat'l University, Korea \\ hmkim@inu.ac.kr
}

\begin{abstract}
This paper aims to develop a centralized optimization framework-based energy management system (EMS), which is used to optimize the day-ahead scheduling of a microgrid $(M G)$ considering vehicle-to-grid service (V2G). Through V2G service, plug-in hybrid electric vehicles (PHEVs) are considered as mobile energy storage systems. The charging/discharging amount of each PHEV during parking time is determined to minimize the operation cost as well as improving the system reliability. Moreover, a mixed integer linear program-based mathematical model is developed for MG operation in both gridconnected and islanded mode considering high penetration of renewable energy sources (RESS) and PHEVs. A test case study considering 10 PHEVs is used to demonstrate the performance of the proposed operation strategy. The numerical results illustrate the effectiveness of $V 2 G$ service on operation of $M G$ in terms of reducing peak load and cost savings in electricity bills.
\end{abstract}

Keywords: Energy management system, mixed integer linear programming, optimal microgrid operation, plug-in hybrid electric vehicle, vehicle-to-grid

\section{Introduction}

Nowadays, the development of microgrids (MGs) has been rapidly increased due to the potential for improving the local reliability and flexibility of the electric power system [1]. MG is defined as a group of renewable distributed sources (RESs), distributed generators (DGs), energy storage system (ESSs), and loads. In normal operation, MG is connected to the utility grid, wherein, MG can exchange surplus/shortage power with external network to maintain the power balance. When MG is disconnected to the utility grid, MG can operate autonomously and fulfill its loads by using its own resources [2]. Distributed ESSs, such as electrochemical batteries, super-capacitors, and flywheels are important applications in MG system, which can shift the surplus power from off-peak intervals to peak intervals for compensation of energy unbalance by charging/discharging mode. As a result, the system reliability can be improved by using ESSs. However, the distributed ESSs require high investment costs [3].

Moreover, the use of electric vehicles (EVs) and plug-in hybrid electric vehicles (PHEVs) have increased rapidly in recent years due to the increase of RESs as well as environmental problems. As a result, the vehicle-to-grid (V2G) service is proposed to use EVs or PHEVs as a cell of ESSs [4]. With considering this service for MG operation, each of PHEV acts as a mobile cell of energy storage system, which can charge/discharge any time during parking time. Therefore, it could be considered as the other alternative for conventional ESS to reduce the investment cost of ESSs. Several operation strategies considering V2G service have been proposed and discussed recently to reduce the total

${ }^{*}$ Corresponding Author 
operation cost. In [5], an MILP-based model for optimal operation of hybrid MG has been employed considering RESs and PHEVs. This model was used to analyze and compare the performance of PHEVs for both conventional AC and hybrid AC/DC MGs. Authors in [6] investigated a demand side management in a commercial building $\mathrm{MG}$ with V2G service to reduce operation cost. In [7], an optimization model for the energy management that considers the uncertainties of demands, EVs, and RESs has been proposed in gridconnected mode. A multi-objective function of the MG system considering V2G service has been developed in [8] for economical operation. Moreover, The EVs/PHEVs are also considered for improving the system reliability. In [9], an optimal control problem has been suggested, wherein, the optimal values of charging/discharging for each EV was determined to optimize MG operations. The evaluation of reliability of the grid has been presented and analyzed in [10] considering V2G operation with various charging/discharging control method. In [11], a decentralized V2G operation scheme has been suggested to support the frequency regulation. However, most of the researches for integration of MG with PHEVs only consider the operation of MG with V2G to improve the system stability or reduce the operation cost in islanded or grid-connected mode. Therefore, this paper focuses on the effectiveness of $\mathrm{V} 2 \mathrm{G}$ service on the MG operation in both grid-connected and islanded modes. The test case study considering 10 PHEVs with various arriving/leaving time is used to demonstrate the performance of the proposed operation strategy. The schedule of each PHEVs is proposed in each operation mode to optimal operation cost as well as reduce load shedding amount.

Moreover, an energy management system (EMS) is proposed to implement their operation strategies. The EMS architectures are grouped into centralized, decentralized, or hybrid EMS [12]. Each of EMS has its advantages and disadvantages. According to [13], centralized energy management is the most cost efficient EMS. Therefore, this paper proposes an operation strategy of MG considering V2G operations based on the centralized optimization framework. In each of operation mode, a comparison between the proposed operation and conventional operation strategies is represented to clarify the effectiveness of V2G service. The paper is organized as follows: Section II represents the proposed operation strategy and an MILP-based mathematical model. Section III describes the numerical results for MG operation considering V2G service. The conclusion of this paper is represented in Section IV.

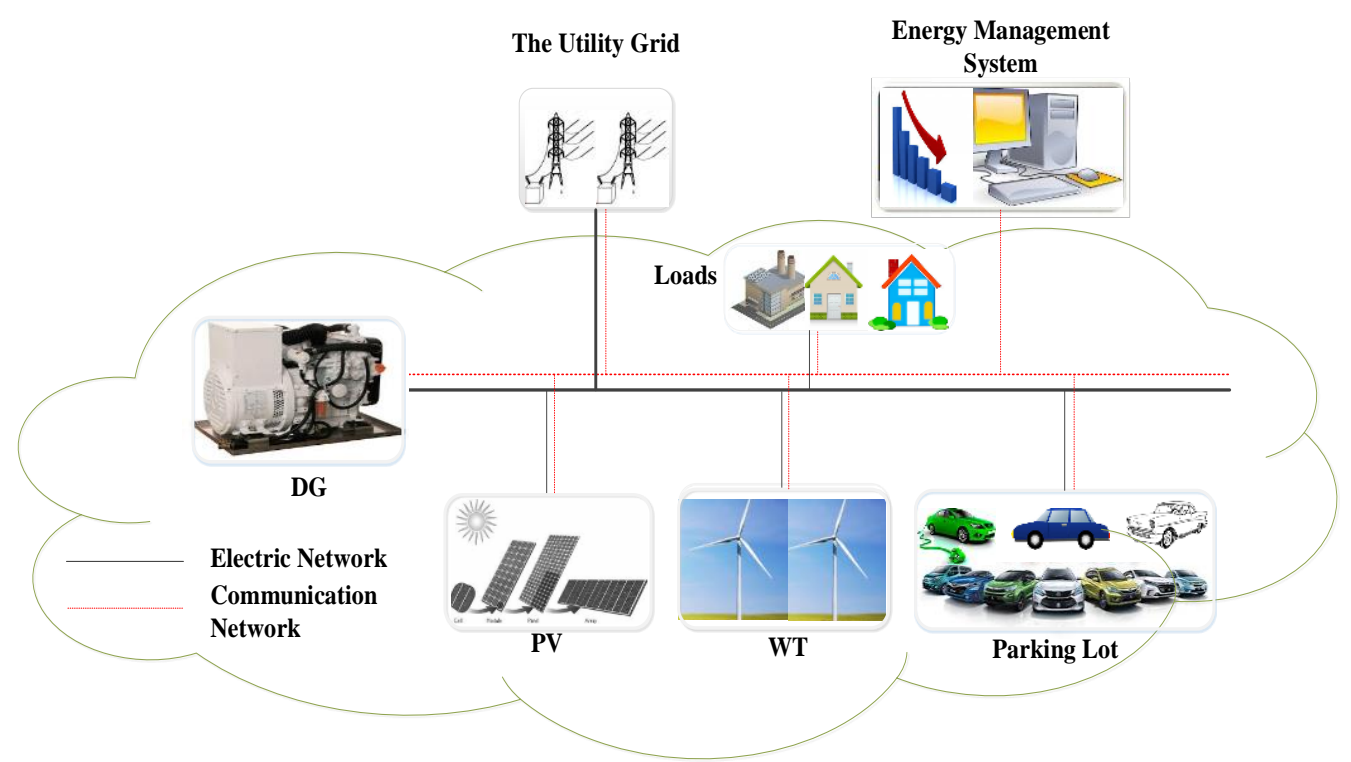

Figure 1. Architecture of Test Microgrid 


\section{System Model}

\subsection{Configuration and components of microgrid}

Figure 1 illustrates an architecture of test MG. It is consisted of fossil-fuel-based generators (diesel generators), RESs (photovoltaics and wind turbines), and parking lot (considering PHEV's battery as an energy storage system), which serves loads. In normal operation, (grid-connected mode), the power balance of the network is provided by exchanging power with the utility grid. On the other hand, in islanded mode, the MG operates without any external network. Therefore, the power balance of the network is maintained by using its own resources. However, in some peak intervals, the demand amount is higher than supply amount. As a result, the load shedding amount should be performed to ensure the power balance in entire network.

In order to implement the proposed operation strategy, a centralized EMS has been developed. In each interval of time, the information of all components in the system are informed to EMS by using communication network. Moreover, an intelligent parking lot management is proposed for parking lot area, wherein, the number of PHEVs and arriving/leaving time are updated and informed to EMS every interval of time. Each PHEV is charged to the required value of SOC before leaving. Based on the receiving information, EMS performs optimization to minimize the total operation cost in entire network.

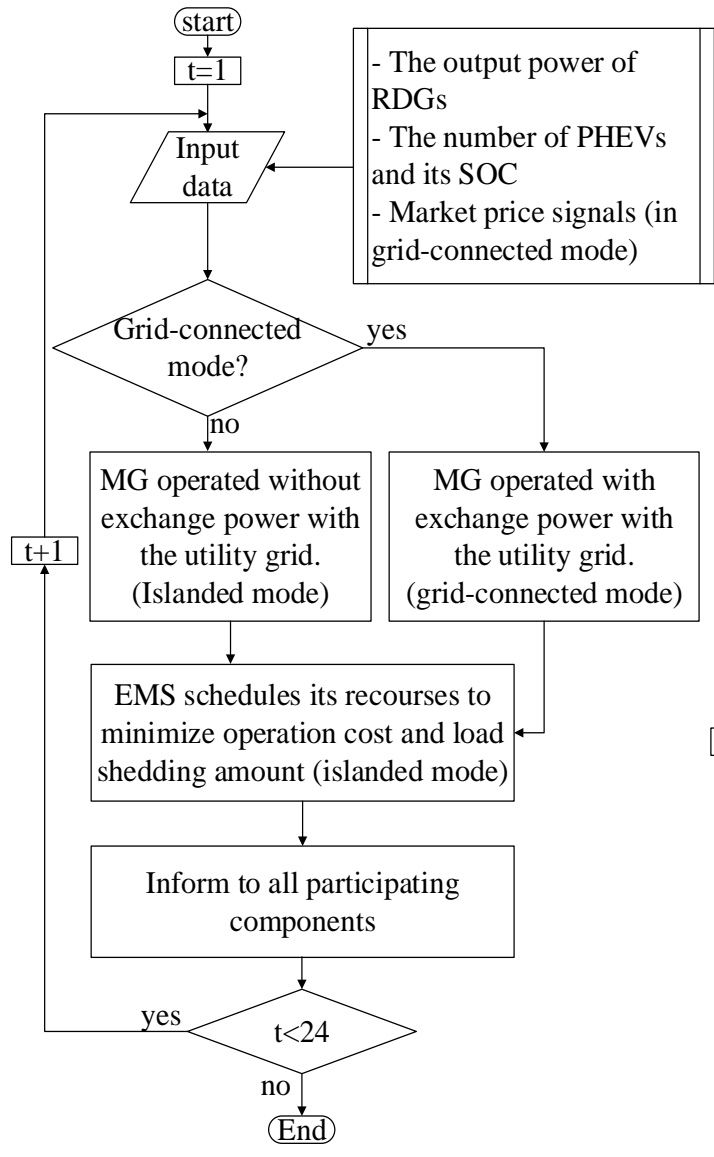

(a)



(b)

Figure 2. Proposed Operation Strategy of Test Microgrid (a). Algorithm for EMS, (b). Algorithm for intelligent parking lot management 


\subsection{System Operation Algorithm}

Figure 2. (a), (b) demonstrate the flow chart related to operation of MG considering V2G service. In Figure 2. (a), the algorithm for EMS is represented while the algorithm for intelligent parking lot management (IPLM) is shown in Figure 2. (b). The proposed operation strategy minimizes the total operation cost as well as reduces amount of load shedding in the MG system (in islanded mode).

Firstly, the output power of RDGs and market price signal along with the information of PHEVs (from IPLM) are informed to EMS via commination network. In grid-connected mode, in order to ensure power balance, MG can export/import power from/to the utility grid while the MG has to perform load shedding for compensating the shortage power. Therefore, EMS schedules its resources to minimize the total operation cost and load shedding amount based on the operation mode and the receiving information. The IPLM, meanwhile, updates the number of PHEVs on parking lot based on the number of arriving/leaving PHEVs. Each PHEV prepares for leaving, which is informed to EMS. The SOC of PHEVs is charged to the required SOC before leaving from parking lot. Finally, EMS informs the optimal operation values to all participating components.

\subsection{Mathematical mModel of MG Operation}

In this section, the MILP-based mathematical formulation of proposed MG is represented. The cost objective function (1) minimizes the operation cost of entire MG. The total operation cost which includes costs associated with fuel consumption, start-up, shutdown, power exchange, and penalty for load shedding.

$$
\begin{aligned}
& \min \sum_{i \in I} \sum_{t \in T}\left(C_{i}^{D G} \cdot P_{i, t}^{D G}+x_{i, t} \cdot C_{i}^{S U_{D G}}+y_{i, t} \cdot C_{i}^{S D_{D G}}\right) \\
& \quad+\sum_{t \in T} v_{t} \cdot\left(P R_{t}^{\text {Buy }} \cdot P_{t}^{\text {Buy }}-P R_{t}^{\text {Sell }} \cdot P_{t}^{\text {Sell }}\right)+\sum_{t \in T}\left(1-v_{t}\right) \cdot\left(\text { pen }_{t}^{\text {Shed }} \cdot P_{t}^{\text {Shed }}\right)
\end{aligned}
$$

Where, the value of $\mathrm{v}_{\mathrm{t}}$ is determined based on the operation mode of MG at interval $\mathrm{t}$, as showed in (2).

$$
v_{t}=\left\{\begin{array}{ll}
1 & \text { in } \text { grid } \text { - connected operation } \\
0 & \text { in islanded operation }
\end{array} \quad \forall t \in T\right.
$$

The first term of (1) determines the operation cost of DG unit that consists of start-up, shut-down costs, and variable cost related to the demand and price of the fuel. The second term represents the cost/profit of buying/selling electricity from/to the utility grid in gridconnected mode. The third term demonstrates the penalty of shedding load in islanded mode.

These constraints of MG operation that are formulated as follows:

$$
\begin{aligned}
& u_{i, t} \cdot P_{i}^{\mathrm{DG}_{\min }} \leq P_{i, t}^{D G} \leq u_{i, t} \cdot P_{i}^{\mathrm{DG}_{\max }} \\
& u_{i, t} \in\{0,1\} \\
& x_{i, t}=\max \left\{\left(u_{i, t}-u_{i, t-1}\right), 0\right\} \\
& y_{i, t}=\max \left\{\left(u_{i, t-1}-u_{i, t}\right), 0\right\}
\end{aligned}
$$

$$
\begin{aligned}
& \forall i \in I, t \in T \\
& \forall i \in I, t \in T \\
& \forall i \in I, t \in T \\
& \forall i \in I, t \in T
\end{aligned}
$$

The Eq. (3), (4) represent the constraint of maximum/minimum output power and on-off mode of each DG unit. Start-up/shut-down status of each DG unit is determined based on on-off mode, as showed in (5), (6), respectively.

The constraint of power balance is given by (7): 


$$
\begin{array}{r}
P_{t}^{P V}+P_{t}^{W T}+\sum_{i \in I} P_{i, t}^{D G}+v_{t} \cdot P_{t}^{\text {Buy }}+\sum_{j \in J} z_{j, t} \cdot P_{j, t}^{B^{-}}+\left(1-v_{t}\right) \cdot P_{t}^{\text {Shed }}=P_{t}^{\text {Load }}+v_{t} \cdot P_{t}^{\text {Sell }}+\sum_{j \in I} z_{j, t} \cdot P_{j, t}^{B^{+}} \\
\forall t \in T
\end{array}
$$

Where, $\mathrm{z}_{\mathrm{i}, \mathrm{t}}$ shows the arrived/leaved status of PHEV $\mathrm{j}$ at interval of time $\mathrm{t}$. the value of $\mathrm{Z}_{\mathrm{i}, \mathrm{t}}$ is determined by Eq. (8).

$z_{j, t}= \begin{cases}1 & \text { PHEVi arrived the parking lot } \\ 0 & \text { PHEVi left the parking lot }\end{cases}$

$$
\forall j \in J, t \in T
$$

The constraints associated with each BESS of PHEV which are given by (9)-(12):

$$
\begin{array}{ll}
0 \leq P_{j, t}^{B^{+}} \leq z_{j, t} \cdot P_{B_{j}}^{M a x} \cdot\left(1-S_{O} C_{j, t-1}^{B}\right) \cdot \frac{1}{1-L_{j}^{B^{+}}} & \forall t \in T \\
0 \leq P_{j, t}^{B^{-}} \leq\left\{\begin{array}{ll}
z_{j, t} \cdot P_{B_{j}}^{M a x} \cdot S O C_{j, t-1}^{B} \cdot\left(1-L_{j}^{B^{-}}\right) & \text {with considering V2G service } \\
0 & \text { without considering V2G service } \\
\operatorname{SOC}_{j, t}^{B}=\operatorname{SOC}_{j, t-1}^{B}-\frac{1}{P_{B_{j}}^{M a x}} \cdot\left(\frac{1}{1-L_{j}^{B^{-}}} \cdot P_{j, t}^{B^{-}}-P_{j, t}^{B^{+}} \cdot\left(1-L_{j}^{B^{+}}\right)\right.
\end{array}\right) & \forall t \in T \\
z_{j, t} \cdot \operatorname{SOC}_{j}^{B_{\min }} \leq \operatorname{SOC}_{j, t}^{B} \leq z_{j, t} \cdot \operatorname{SOC}_{j}^{B_{\max }} & \forall t \in T
\end{array}
$$

Maximum charging and discharging constraints of each PHEV BESS are represented by (9), (10), respectively. The SOC is continuously updated according to Eq. (11) in each interval of time. Constrain (12) denotes operated bounds of PHEV BESS.

The constraint (13) enforces the minimum SOC of PHEV BESS before leaving.

$$
\operatorname{SOC}_{j, t_{\text {leav. }}}^{B} \geq S O C_{j}^{B_{\text {req. }}} \quad \forall j \in J
$$

\section{Numerical Results}

\subsection{Input Data}

In this study, the proposed strategy operation is implemented for a test MG, which is consisted of 1 PV, 1 WT, 1 DG, 10 PHEVs, and loads, as shown in Figure 1. The proposed model is formulated for a 24-hour scheduling horizon. Each time of interval is set to be 1 hour. The buying and selling prices along with generation cost for DG are depicted in Figure 3(a) while RDG outputs, and load profiles are given in Figures 3(b), (c), respectively. Minimum/maximum generation and start-up/shutdown cost along with generation cost of DG unit are tabulated in Table 1. Parameters related to BESSs of PHEVs and the assumed arriving/leaving time are shown in Table 2.

The proposed operation strategy is applied for test MG in both grid-connected and islanded modes. Moreover, the effectiveness of V2G service are also shown under difference operation modes. The mentioned models have been coded and solved by Java software using IBM ILOG CPLEX solver [14]. 


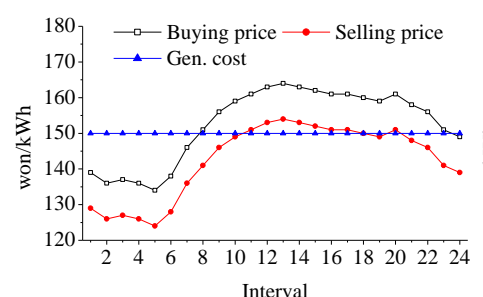

(a)

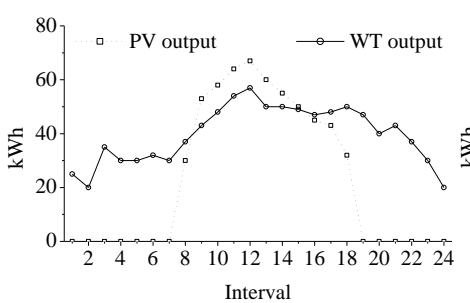

(b)

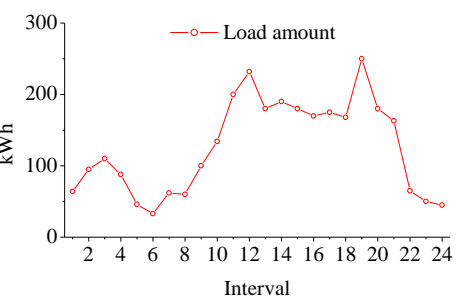

(c)

Figure 3. (a). Market Price Signals and Generation cost

(b). Output Power of PV and WT

(c). Load profiles

Table 1. Diesel Generator Parameters

\begin{tabular}{|l|c|}
\hline \multicolumn{1}{|c|}{ Parameters } & DG \\
\hline Min. (kWh) & 0 \\
\hline Max. (kWh) & 80 \\
\hline Start-up cost (KRW) & 300 \\
\hline Shut-down cost (KRW) & 200 \\
\hline Operation cost (KRW/kWh) & 150 \\
\hline
\end{tabular}

Table 2. PHEVs' BESS Parameters

\begin{tabular}{|c|c|c|c|c|c|c|c|c|}
\hline \multirow{2}{*}{ PHEVs } & \multicolumn{7}{|c|}{ BESS Parameters } \\
\cline { 2 - 9 } & $\begin{array}{c}\text { Min. } \\
(\mathbf{k W h})\end{array}$ & $\begin{array}{c}\text { Max. } \\
(\mathbf{k W h})\end{array}$ & $\begin{array}{c}\text { Ini. } \\
(\mathbf{k W h})\end{array}$ & $\begin{array}{c}\text { SOC_leav. } \\
(\mathbf{k W h})\end{array}$ & $\begin{array}{c}\text { Loss_c } \\
(\boldsymbol{\%})\end{array}$ & $\begin{array}{c}\text { Loss_d } \\
(\boldsymbol{\%})\end{array}$ & $\begin{array}{c}\text { Arri. Time } \\
(\text { hour })\end{array}$ & $\begin{array}{c}\text { Leav. Time } \\
(\text { hour })\end{array}$ \\
\hline 1 & 0 & 10 & 3 & 8 & 2 & 2 & $1-12$ & $12-24$ \\
\hline 2 & 0 & 15 & 5 & 10 & 2 & 2 & $8-19$ & $1-8,19-24$ \\
\hline 3 & 5 & 10 & 6 & 10 & 2 & 2 & $1-12$ & $12-24$ \\
\hline 4 & 0 & 5 & 2 & 5 & 2 & 2 & $1-8$ & $8-24$ \\
\hline 5 & 0 & 5 & 2 & 5 & 2 & 2 & $1-8$ & $8-24$ \\
\hline 6 & 5 & 10 & 7 & 8 & 2 & 2 & $1-22$ & $22-24$ \\
\hline 7 & 5 & 10 & 7 & 8 & 2 & 2 & $18-24$ & $1-18$ \\
\hline 8 & 10 & 20 & 15 & 20 & 2 & 2 & $8-19$ & $1-8,19-24$ \\
\hline 9 & 0 & 15 & 5 & 15 & 2 & 2 & $8-19$ & $1-8,19-24$ \\
\hline 10 & 0 & 10 & 5 & 8 & 2 & 2 & $10-20$ & $1-10,20-24$ \\
\hline
\end{tabular}

\subsection{MG Operation in Grid-Connected Mode}

In this mode, MG can export/import power from/to the utility grid. The export/import power is determined based on the comparison between the market prices and generation cost to minimize the operation cost of entire MG. In conventional operation strategy (without V2G service), the PHEVs is considered as loads, which are charged to the required value of SOCs before leaving from parking lot. The BESSs is charged with cheaper resources based on the arriving time. On the other hand, with considering V2G service, the PHEV's BESSs can charge/discharge to minimize the MG operation cost during the parking time. The total amount of charging/discharging power for both cases are summarized in Figures 4. (a), (b). By focusing on Figures 4, it can be seen that the BESSs is used to charge 


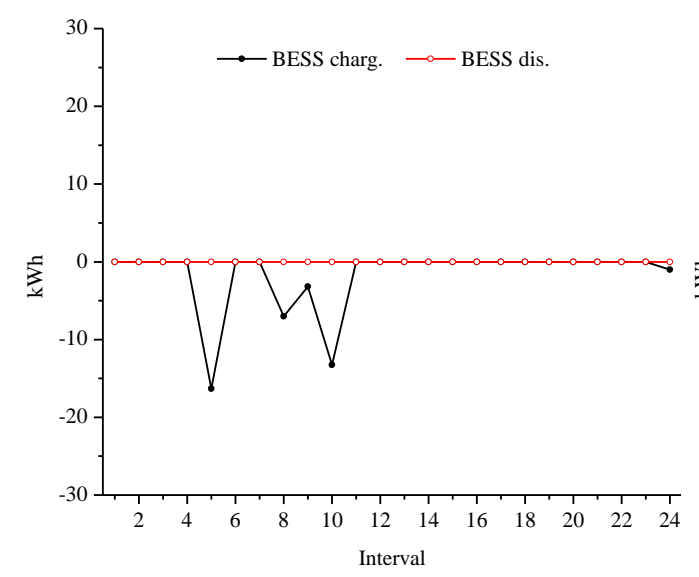

(a)

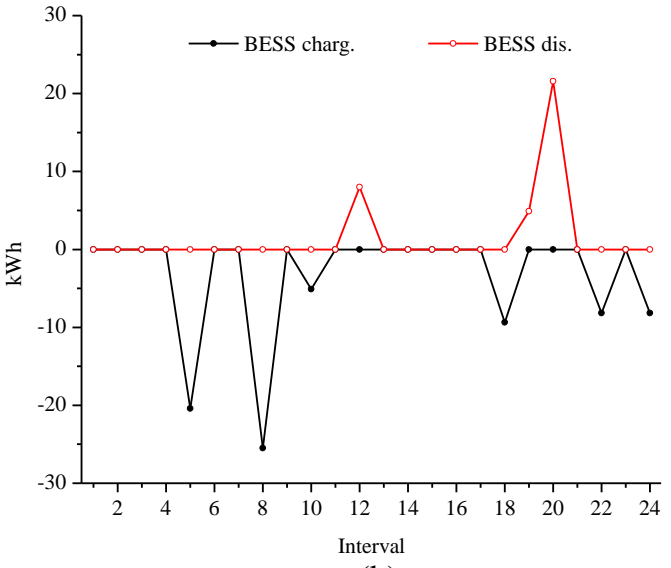

(b)

Figure 4. Total BESS Charging/Discharging Amount

(a). Without V2G service

(b). With V2G service
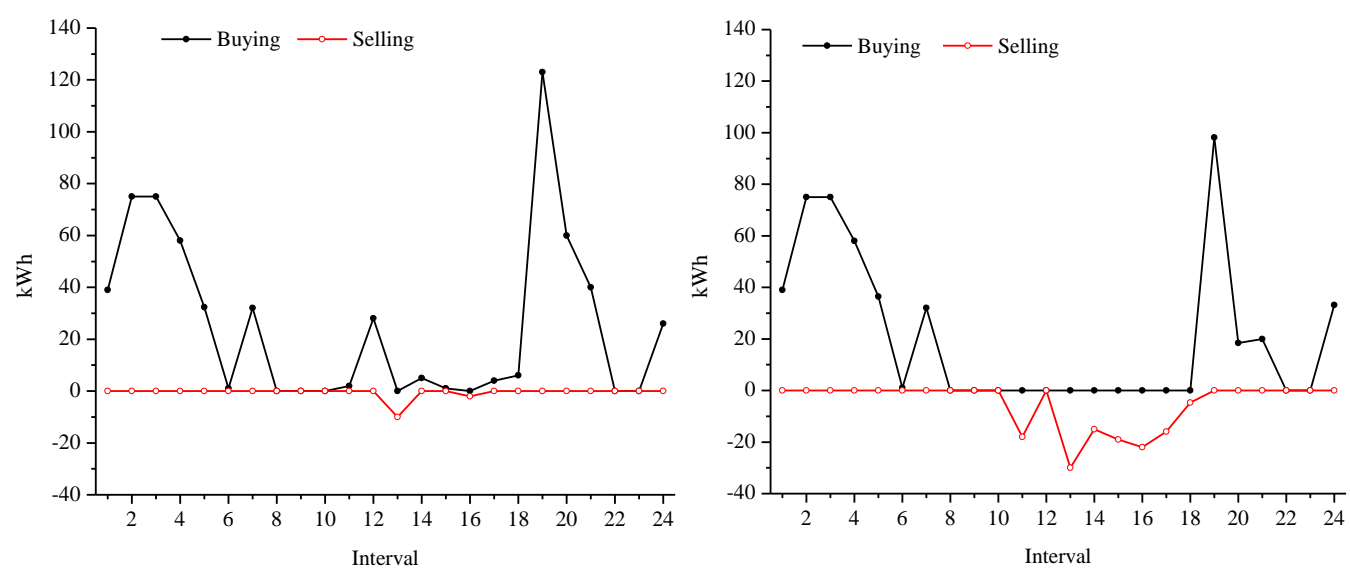

Figure 5. Total buying/selling amount

(a). Without V2G service

(b). With V2G service

with the cheapest resource (at interval 5-8) and discharge as supplies instead of expensive resources (interval 12,19-21). Similarly, total amount of buying/selling power for both cases has been depicted in Figure 5. By applying the proposed strategy considering V2G service, the amount of buying power is decreased in peak-hour time $(12,19-21)$ while the amount of selling power is increased to maximize the profit due to economical operation.

Figures 6. (a)- (j) illustrate the SOC of each PHEV's BESS in grid-connected mode. With the proposed operation strategy, each BESS is considered as a small energy storage system during parking time. It can be seen that the SOC of PHEVs, which is always charged to the required 

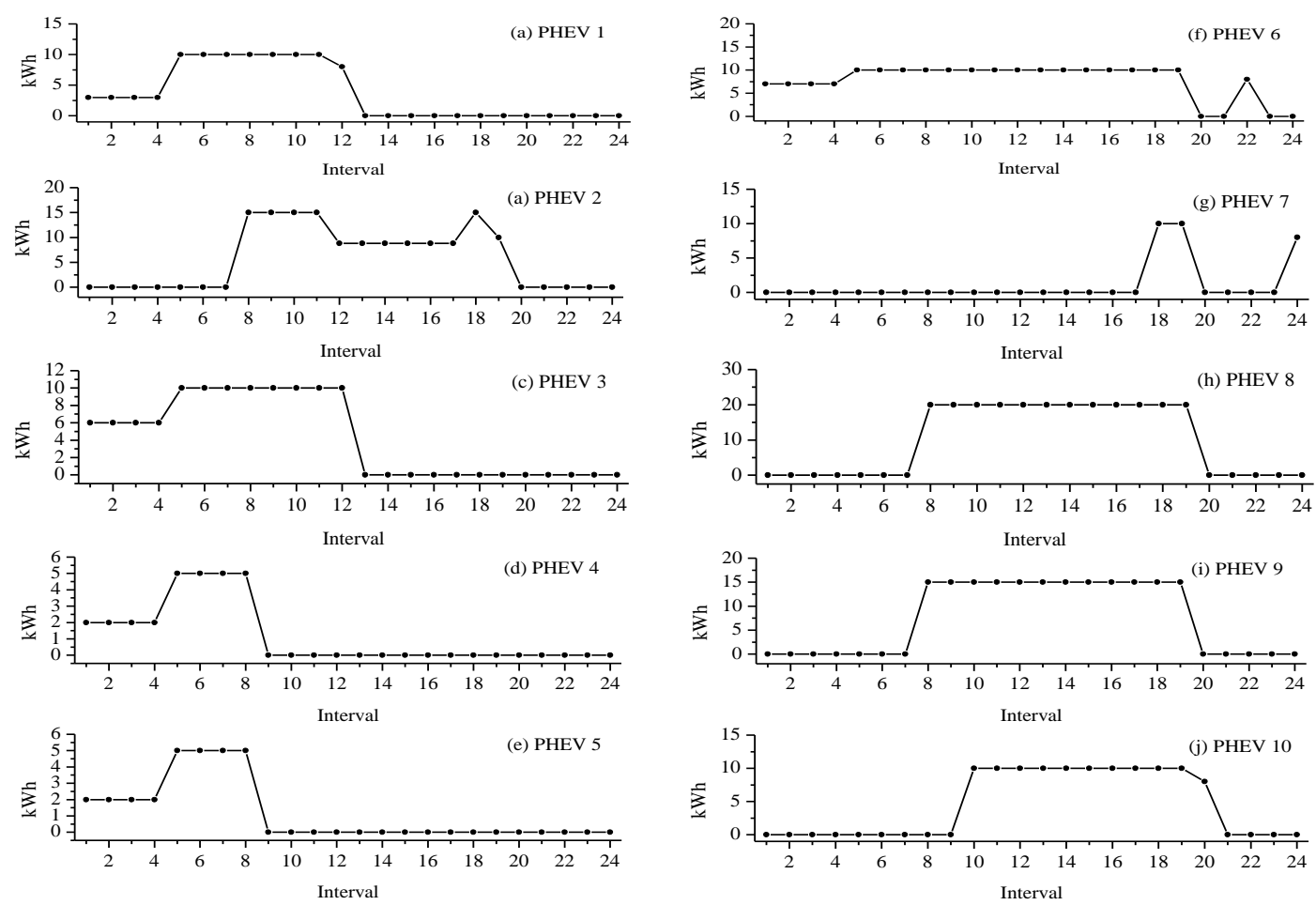

Figure 6. SOC of each PHEV's BESS in Grid-Connected Mode

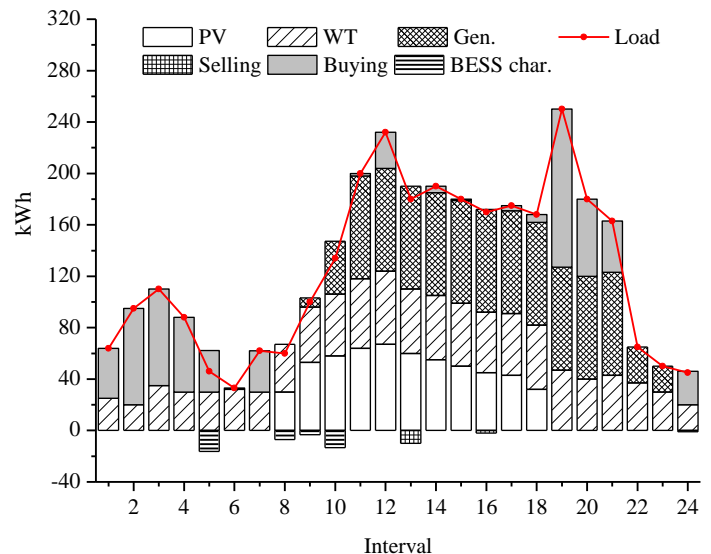

(a)

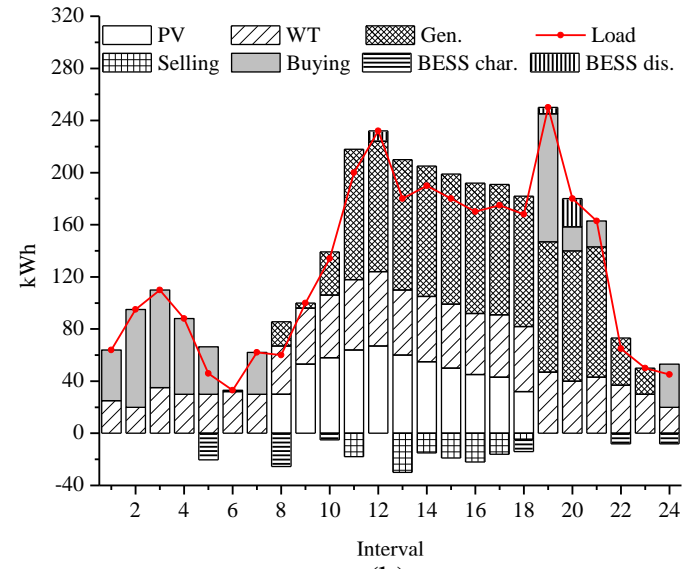

(b)

Figure 7. MG operation in grid-connected mode

(a). Without V2G service

(b). With V2G service

value before leaving time. Moreover, based on the arriving time, the charging/discharging of BESSs are determined to minimize the operation cost. In this case, the PHEV 1, 2, 6, 7, 10 are used to operate in the MG system.

Figure 7. (a), (b) demonstrate the day-ahead schedules of all MG's resources; namely output power of WT, PV, diesel generator, total amount of charging/discharging power of PHEVs and amount of buying/selling power from/to the utility grid without V2G service and with V2G service, respectively. In Figure 7. (b), it can be figured out from the results the amount of buying power is reduced by using PHEVs, which charge with surplus power and discharge to reduce the amount of deficit power. 


\subsection{MG Operation in Islanded Mode}

In this section, the effectiveness of V2G service is represented for the MG operation in islanded mode. The load shedding amount is determined with considering the $\mathrm{V} 2 \mathrm{G}$ service and without the V2G service. Moreover, the SOC of all PHEVs are updated at each interval of time during the parking time.

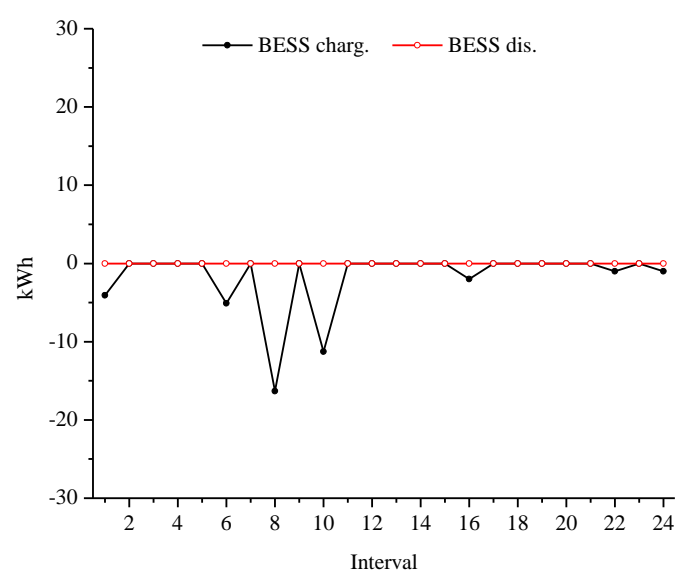

(a)



(b)

Figure 8. Total PHEV's BESS charging/discharging in MG

(a). Without V2G service

(b). With V2G service

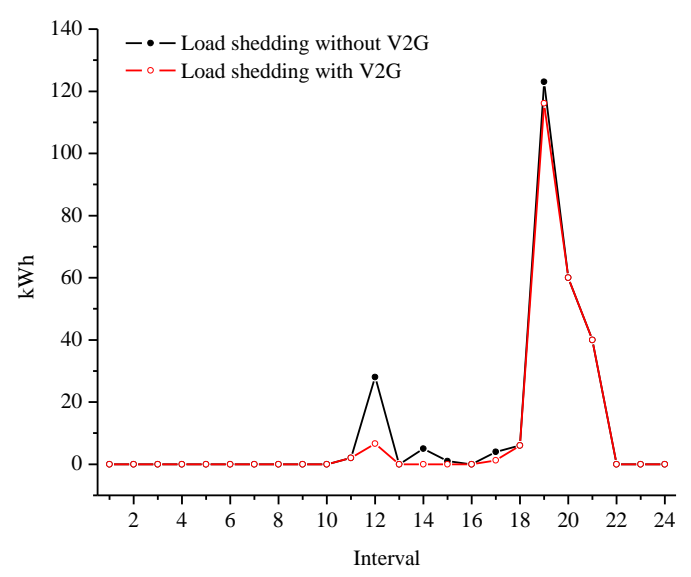

Figure 9. Load Shedding Amount in Islanded Mode

In islanded model, MG is operated without the utility grid. Therefore, MG has to schedule its resources to fulfill its loads without external resources. Fig. 8 indicates that the total amount of PHEV's BESS charging/discharging. Without V2G service, the BESSs charge only during the parking time. The charging amount depends on the number of PHEVs as shown in Figure 8. (a). However, with V2G service, the BESSs can charge/discharge many time due to economical operation. It can be obtained from Figure 8. (b), the surplus power is charged (interval 1,2,8-11) and discharged to reduce shortage amount in the system. The increase of charging/discharging amount can reduce the load shedding amount, as shown in Figure 9. The surplus power is shifted from off-peak intervals to peak intervals by using PHEV's BESS. The decrease of load shedding amount is depended on the capacity of BESS and parking time of each PHEV. 
Figures 10. (a)- (j) demonstrate the SOC of each PHEV's BESS in islanded mode. The SOC of BESSs are updated each interval of time based on charging/discharging amount. During parking time, PHEVs is scheduled by EMS for minimizing the operation cost as

well as reducing load shedding amount. The EMS also ensures that the SOC of PHEVs is charged to required values before leaving from parking lot. Based on the parking time, the EMS decides the number of PHEVs, which are used as a cell of energy storage system in the MG system. In this case, the PHEV 1, 2, 6, 7, 10 are used for MG operation.

Finally, the day-ahead scheduling of MG operation is shown for islanded mode in Figure 11. The schedules of all components without V2G service is represented in Figure 11. (a). It can be seen that the amount of load shedding has been performed to ensure the power
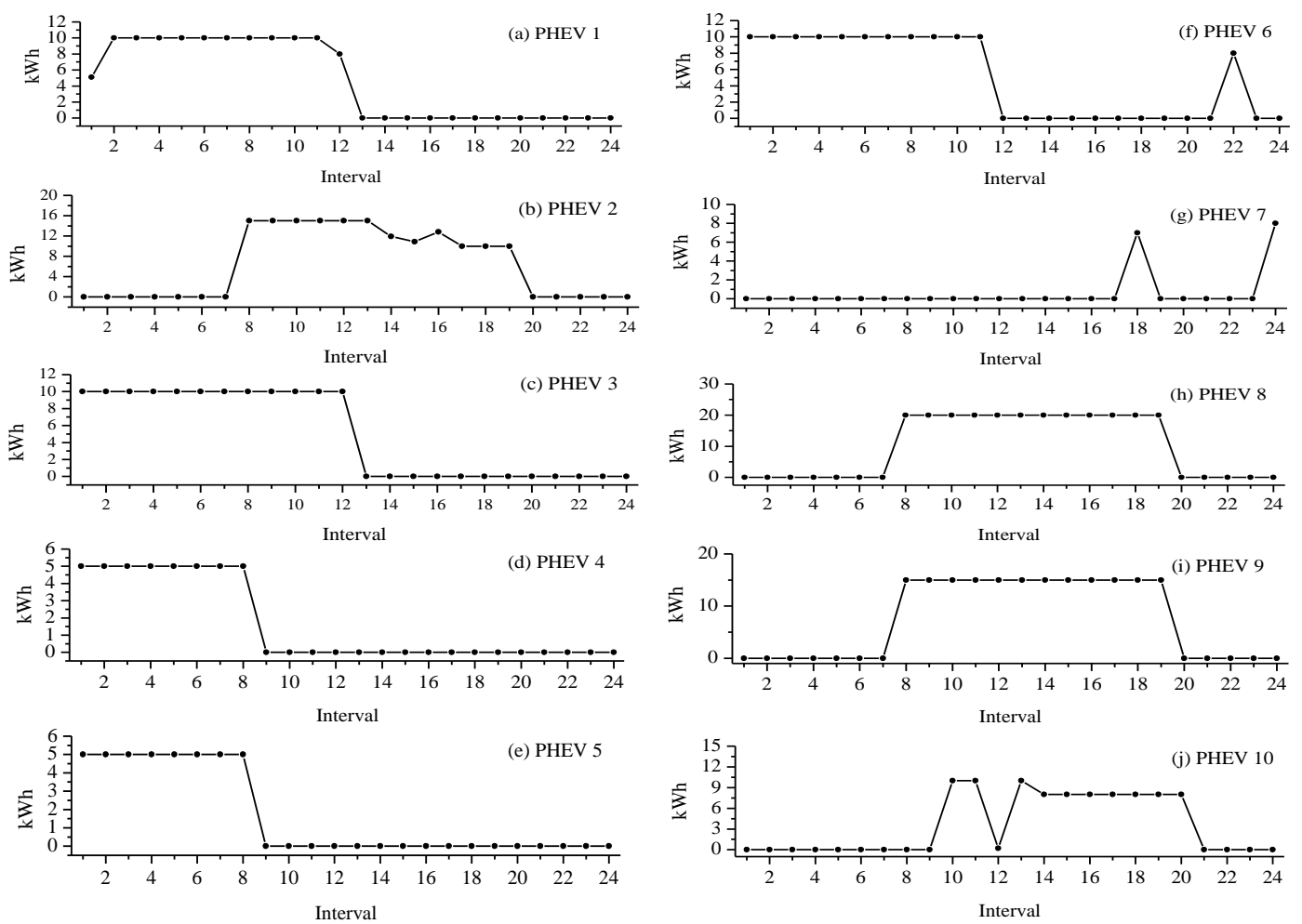

Figure 10. SOC of each PHEV in Islanded Mode



(a)

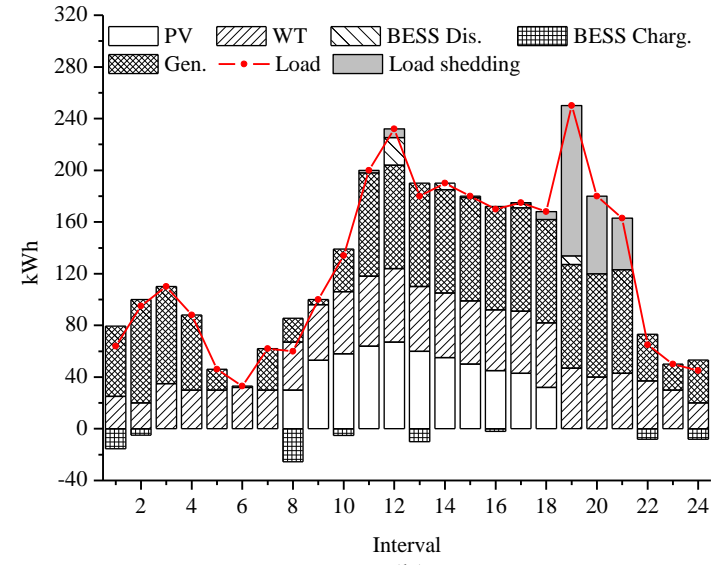

(b)

Figure 11. MG Operation in islanded mode

(a). Without V2G service

(b). With V2G service 
balance in the MG system. In order to reduce the load shedding amount, the MG system is operated with V2G service. In this case, the day-ahead scheduling of MG system is shown in Figure 11. (b). It can observed that the load shedding amount has been decreased by using the amount of discharging power from PHEV's BESS.

\section{Conclusion}

In this paper, an operation strategy has been proposed to minimize the total operation cost of entire network considering V2G service. The effectiveness of V2G service have been represented for both grid-connected and islanded modes. In grid-connected mode, the PHEVs are charged and discharged with the effective prices to reduce the operation costs. Therefore, the import power from the utility grid can be reduced by using the service. Moreover, the load shedding amount is also decreased in islanded mode. Finally, it can be concluded from the numerical results that the system can reduce the operation cost and the amount of load shedding by using proposed operation strategy considering V2G service.

\section{Nomenclature}

$\begin{array}{ll}\text { Sets } & \text { Set of time intervals. } \\ T & \text { Set of diesel generations. } \\ J & \text { Set of PHEV s. } \\ \text { Constants } & \text { Production cost of DG unit i. } \\ C_{i}^{D G} & \text { Start-up cost of DG unit i. } \\ C_{i}^{S U_{D G}} & \text { Shut-down cost of DG unit i. } \\ C_{i}^{S D_{D G}} & \text { Buying/selling price at t. } \\ P R_{t}^{\text {Buy }}, P R_{t}^{\text {Sell }} & \text { Forecasted output of PV cell \& wind turbine at t. } \\ P_{t}^{P V}, P_{t}^{W T} & \text { Load amount at t. } \\ P_{t}^{\text {Load }} & \text { Penalty for load shedding amount at t. } \\ \text { pen }_{t}^{\text {shed }} & \text { Losses for charging/discharging of PHEV BESS unit j. } \\ L_{j}^{B^{+}}, L_{j}^{B^{-}} & \text {Capacity of PHEV BESS unit j. } \\ P_{B_{j}}^{\text {Max }} & \text { Required state of charge of PHEV BESS unit j before leaving. } \\ S O C_{j}^{B_{\text {req. }}} & \end{array}$

Variables

$u_{i, t}$

On or off mode of DG unit $i$ at $t$.

$P_{i, t}^{D G}$

Generation amount of DG unit $\mathrm{i}$ at $\mathrm{t}$.

$P_{t}^{\text {Buy }}, P_{t}^{\text {Sell }}$

Buying/selling power from/to the utility grid at $t$.

$P_{j, t}^{B^{+}}, P_{j, t}^{B^{-}}$

Charging/discharging power of PHEV BESS unit $\mathrm{j}$ at $\mathrm{t}$.

$P_{t}^{\text {Shed }}$

Load shedding amount at $\mathrm{t}$.

$\operatorname{SOC}_{j, t}^{B}$

State of charge of PHEV BESS unit $\mathrm{j}$ at $\mathrm{t}$.

$\operatorname{SOC}_{j, t_{\text {leav }}}^{B}$

State of charge of PHEV BESS unit $\mathrm{j}$ at leaving time. 


\section{Acknowledgments}

This work was supported by the Korea Institute of Energy Technology Evaluation and Planning (KETEP) and the Ministry of Trade, Industry \& Energy (MOTIE) of the Republic of Korea (No. 20151210200080).

\section{References}

[1] X. Fang, S. Misra, G. Xue, D. Yang, "Smart grid-The new and improved power grid: A survey,” IEEE Commun. Surv. Tutor., vol. 14, no. 4, (2012), pp. 944-980.

[2] H.-M. Kim, Y. Lim, and T. Kinoshita, "An intelligent multiagent system for autonomous microgrid operation," Energies, vol. 5, no. 9, (2012), Sept., pp. 3347-3362.

[3] S. Teleke, M. E. Baran, S. Bhattacharya, and A. Q. Huang, "Rule-based control of battery energy storage for dispatching intermittent renewable sources," IEEE Trans. on Sustainable Energy, vol. 1, Sept. (2010), pp. $117-124$.

[4] S. Han, S. Han, and K. Sezaki, "Development of an optimal vehicle-to grid aggregator for frequency regulation," IEEE Trans. on Smart Grid, vol. 1, no. 1, June (2010), pp. 65-72.

[5] P. T. Baboli, S. Bahramara, M. P. Moghaddam, and M. R. Haghifam, "A mixed-integer linear model for optimal operation of hybrid AC-DC microgrid considering Renewable Energy Resources and PHEVs," In PowerTech, IEEE Eindhoven, Jun. (2015), pp. 1-5.

[6] Y. Wang, B. Wang, C. C. Chu, H. Pota, and R. Gadh, "Energy management for a commercial building microgrid with stationary and mobile battery storage," Energy and Buildings, vol. 116, (2016), pp. 141150.

[7] E. Mortaz and J. Valenzuela, "Microgrid energy scheduling using storage from electric vehicles," Electric Power Systems Research, Nov. (2016).

[8] H. Liu, Y. Ji, H. Zhuang, and H. Wu, "Multi-objective dynamic economic dispatch of microgrid systems including vehicle-to-grid," Energies, vol. 8, no. 5, (2015), pp. 4476-4495.

[9] M. Musio, M. Porru, A. Serpi, I. Marongiu, and A. Damiano, "Optimal electric vehicle charging strategy for energy management in microgrids," In Electric Vehicle Conference (IEVC), IEEE International, Dec. (2014), pp. 1-8.

[10] M. Jun and A. J. Markel, "Simulation and analysis of vehicle-to-Grid operations in microgrid," In IEEE Power and Energy Society General Meeting, Jul. (2012), pp. 1-5.

[11] S. W. Kim, Y. G. Jin, Y. H. Song, Y. T. and Yoon, "Decentralized Vehicle-to-Grid Design for Frequency Regulation within Price-based Operation,” J. Electr. Eng. Technol, vol. 10, no. 3, (2015), pp. 1335-1341.

[12] V. H. Bui, A. Hussain, and H. M. Kim, "A multiagent-based hierarchical energy management strategy for multi-microgrids considering adjustable power and demand response," IEEE Trans. on Smart Grid, accepted, (2016), pp. 1-10.

[13] A. Hussain, V. H. Bui, and H. M. Kim, "A resilient and privacy-preserving energy management strategy for networked microgrids," IEEE Trans. on Smart Grid, accepted, (2016), pp. 1-12.

[14] IBM ILOG CPLEX V12.1 User's Manual for CPLEX 2009, CPLEX Division, ILOG, Incline Village, NV, USA, (2009).

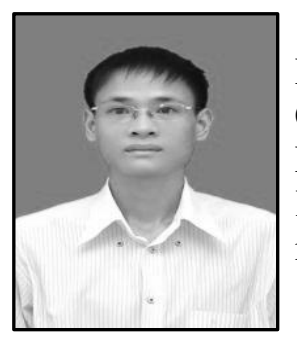

Van-Hai Bui, received B.S. degree in Electrical Engineering from Hanoi University of Science and Technology, Vietnam in 2013. Currently, he is a combined Master and Ph. D. student in the Department of Electrical Engineering, Incheon National University, Korea. His research interests include microgrid operation and energy management system (EMS).

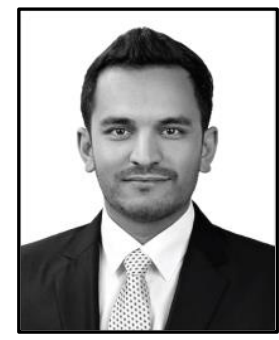

Akhtar Hussain, received his B.E degree in Telecommunications from National University of Sciences and Technology (NUST) Pakistan in 2011 and M.S in Electrical Power Systems from Department of Electrical \& Electronics Engineering, Myongji University, Korea, in 2014. He worked for SANION, Korea from Jan 2014 to May 2015. Currently he is a Ph.D. student in Power \& Renewable Energy Lab, Department of Electrical Engineering, 
Incheon National University, Korea. His research interests are power system automation and protection, smart grids, operation of microgrids, and energy management in microgrids.

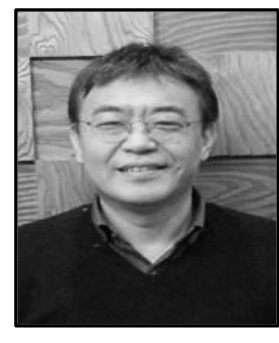

Hak-Man Kim, received his first Ph.D. degree in Electrical Engineering from Sungkyunkwan University, Korea in 1998 and received his second $\mathrm{Ph}$. D. degree in Information Sciences from Tohoku University, Japan, in 2011, respectively. He worked for Korea Electrotechnology Research Institute (KERI), Korea from Oct. 1996 to Feb. 2008. Currently, he is a professor in the Department of Electrical Engineering, Incheon National University, Korea. His research interests include microgrid operation \& control and DC power systems. 
International Journal of Control and Automation Vol.10, No.3 (2017) 